\title{
Cloze probability and completion norms for 498 sentences: Behavioral and neural validation using event-related potentials
}

\author{
CADY K. BLOCK \\ University of Alabama, Birmingham, Alabama \\ AND \\ Carryl L. BAldWiN \\ George Mason University, Fairfax, Virginia
}

\begin{abstract}
Three decades after their publication, Bloom and Fischler's (1980) sentence completion norms continue to demonstrate widespread utility. The aim of the present study was to extend this contribution by expanding the existing database of high-constraint, high cloze probability sentences. Using the criteria established by Bloom and Fischler, we constructed 398 new sentences and presented these along with 100 sentences from their original list to be normed using a sample of 400 participants. Of the 498 sentences presented, 400 met criteria for high cloze probability - that is, .67 or higher probability of being completed by a specific single word. Of these, 321 sentences were from the new set and an additional 79 were from Bloom and Fischler's set. A high degree of correspondence was observed between responses obtained by Bloom and Fischler for their high-constraint set. A second experiment utilized an N400 event-related potential paradigm to provide further validation of the contextual constraint for the newly generated set. As expected, N400 amplitude was greater for sentences that violated contextual expectancy by ending in a word other than the newly established completion norm. Sentence completion norms are frequently used in cognitive research, and this larger database of high cloze probability sentences is expected to be of benefit to the research community for many years to come. The full set of stimuli and sentence completion norms from this study may be downloaded from http://brm.psychonomic-journals.org/ content/supplemental.
\end{abstract}

Language discourse requires the ability to utilize context to disambiguate word meanings and to anticipate sentence endings to facilitate comprehension, preparation for turn taking, and appropriate response. Simply put, an expectation of what words or sentences are to come makes it easier to communicate. Contextually based word expectancies in normal conversation facilitate turn taking in conversational exchange, allow us to finish another person's sentences (Van Berkum, Brown, Zwitserlood, Kooijman, \& Hagoort, 2005), and contribute to our understanding and appreciation of humor (Coulson, Urbach, $\&$ Kutas, 2006).

People's ability to use context during speech processing, and their response to violations of contextual expectancy, have been used extensively in the literature as a means of examining a number of cognitive processes (Bloom \& Fischler, 1980; Schwanenflugel \& LaCount, 1988; Taylor, 1953; Traxler \& Foss, 2000; Van Berkum et al., 2005). For example, Federmeier and colleagues have used contextual expectancies and violations in speech processing tasks to investigate potential age-related changes in semantic memory organization (Federmeier, McLennan, De
Ochoa, \& Kutas, 2002), and to differentiate the relative contributions of acoustic and contextual level factors associated with age-related changes in speech processing (Federmeier, van Petten, Schwartz, \& Kutas, 2003; StineMorrow, Miller, \& Nevin, 1999).

Of paramount importance to experiments aimed at examining the influence of context on language-based tasks is the existence of a set of verbal stimuli with wellestablished "normed" properties (Bradshaw, 1984). Large sample normative data must be established in order to systematically examine the influence of other factors (i.e., age, stimulus salience, and syntax) on the relative importance of context to the performance of language tasks.

In 1980, Bloom and Fischler published a set of completion norms for sentences varying from low to high context. Since that time, their stimuli have been used extensively, resulting in at least 150 peer-reviewed journal citations based on counts from the Social Science Citation Index and Google Scholar alone, and have been referenced in numerous other books, book chapters, theses, dissertations, and articles. The impact of their work stems from a recognition of the important role that context plays in

C. L. Baldwin, cbaldwi4@gmu.edu 
speech processing and the need for a standardized stimulus set to facilitate comparisons between investigations.

Among the many contributions that the Bloom and Fischler (1980) stimuli have made possible is the establishment of a now well-documented semantically based event-related potential (ERP) component. Using sentences from Bloom and Fischler's completion norm list, Kutas and Hillyard (1984) confirmed the existence of a negative wave deflection ERP component peaking around $400 \mathrm{msec}$ (termed the N400), whose amplitude is inversely correlated with expectancy for the final word in a sentence. These results provided conclusive evidence for their observations of a semantically based ERP component published a few years earlier (Kutas \& Hillyard, 1980, 1983).

The N400 response has since been used extensively to examine the influence of context in both reading and speech processing. The N400 amplitude varies with semantic expectancy, or the extent to which a word is semantically primed. Ninety percent of the variance found in the N400 amplitude can be predicted by the fit of a word within a given context (Coulson et al., 2006). Kutas and Hillyard (1984) observed reduced amplitudes to more predictable words and increased amplitudes to less predictable words. This observation has been replicated in numerous investigations using both text and speech stimuli (Federmeier et al., 2002; Van Berkum et al., 2005).

Despite the continued utility of the Bloom and Fischler (1980) stimuli, their validity for use with current generation participants and among different age groups has been questioned (Lahar, Tun, \& Wingfield, 2004). Lahar and colleagues examined the extent to which today's young adults, born on average two decades later than those examined by Bloom and Fischer, would generate the same completion norms. Furthermore, Lahar and colleagues questioned the extent to which completion norms for the Bloom and Fischler stimuli are applicable to adults from different age groups. Lahar and colleagues documented a high degree of consistency between their results and those of Bloom and Fischler's completion norms for high cloze probability stimuli-both across generations of young adults and between responses obtained from individuals ranging in age from young and middle-aged to young-old and old-old. These results, in addition to their continued use, attest to the continued utility of the Bloom and Fischler stimuli.

At the same time, a number of current issues warranting further examination require a more extensive set of sentences than is currently available in the Bloom and Fischler (1980) completion norms. For example, in order to investigate the relative impact of various acoustic factors versus cognitive factors, a larger set of high-constraint sentences would be useful. In order to obtain a robust N400 component, between 60 and 80 sentence stimuli per condition are generally required. Unless sentences are to be repeated several times, the current set of validated completion norms restricts the number of factors that can be compared in any given participant population. Bloom and Fischler's highconstraint sentence (low uncertainty, or LU) list contains only 125 sentences with cloze probabilities greater than or equal to .67. Their variable uncertainty (VU) list contains an additional 37 sentences that would meet our definition of high cloze probability. But these sentences, as the name implies, have been found to be less reliably high cloze probability sentences (Lahar et al., 2004).

The purpose of the present study was to construct a large set of high-constraint sentences, verify their cloze probability, and establish completion norms for an additional database of sentences using the methodology established by Bloom and Fischler (1980), who noted a relative lack of standardized language materials. In line with their aims, it is our hope that the sentence norms presented here, which include a substantial number of new high cloze sentences, will be of use to other researchers interested in the contextual effects of processing words in sentences. This standardized set will provide a consistent resource that will allow reliable comparison across studies, in addition to being a valuable tool for research in such areas as understanding the relative intelligibility of various forms of computerized speech, age-related changes in language processing, and various forms of electrophysiological, neuropsychologi$\mathrm{cal}$, and neuroimaging language-based research.

\section{Using the Cloze Method to Examine Context-Based Word Prediction}

The cloze method was developed to examine contextbased word prediction (Taylor, 1953) - that is, the predictability of the sentence-final word on the basis of the prior context of the sentence. Language passages are constructed with missing elements, and the reader must predict and complete the unfinished sentences with the word or words (called the target or primary response [Bloom \& Fischler, 1980]) he or she judges to be the most appropriate, on the basis of the accumulated preceding context. The proportion of participants who give a certain response to finish an incomplete sentence or passage may then be measured. This proportion is called the cloze value or cloze probability (Kutas \& Hillyard, 1984) and may be low, moderate, or high. High cloze probability is defined in Coulson et al. (2006) as $40 \%$ or higher. However, Bloom and Fischler utilized a much more stringent criterion of $90 \%$ or above for high cloze probability. For the purposes of the present study, low cloze was defined as $0 \%-33 \%$, medium cloze as $34 \%-66 \%$, and high cloze as $67 \%-100 \%$.

In their 1980 study, Bloom and Fischler developed a set of standardized sentence completion norms using the cloze method and reported responses and values for each sentence. The researchers used two different sets of sentence contexts, the first of which was dubbed the VU set, which produced a large range of probabilities for the primary response. These ranged from high-constraint sentence contexts that would produce very likely responses (or what they called "dominant") to contexts with more variability in the completion responses. Their second set, or LU set, involved only sentence contexts with high cloze probability responses, although it still allowed for some alternative responses. They found a greater proportion of very probable primary responses in the $\mathrm{LU}$ than in the $\mathrm{VU}$ set, and these norms are considered to be benchmark sentence sets and are used in studies with diverse population samples ranging from children to adults to people with Alzheimer's disease (Lahar et al., 2004). 


\section{The Present Study}

The present study consists of two experiments. The first experiment utilized the cloze method to examine contextbased sentence completion by prediction of a missing final word. A total of 398 newly created sentences were interspersed with 100 selected high-constraint sentences from Bloom and Fischler's (1980) lists and were administered to a large sample of participants. Experiment 1 was designed to obtain cloze probabilities for the new sentences and to validate the selection of original sentences from Bloom and Fischler. Experiment 2 was designed to extend the behavioral validation by using the N400 ERP component as a neurophysiological index of semantic context effects. The aim of both these experiments was to develop and validate a large database of high-constraint sentences.

\section{EXPERIMENT 1}

\section{Method}

Participants. Four hundred individuals from psychology classes at a Virginia university voluntarily participated in this investigation. Thirty-three participants $(8 \%)$ completed less than $50 \%$ of the sentences or had a response style obviously inappropriate for the purposes of the present study (e.g., putting the same word for every answer; giving prose or long phrases, rather than single words as directed). These individuals were dropped from the subsequent analysis, resulting in a sample of 377 participants (18-51 years of age; $M=20.5$ years, $S D=4.26$ ) included in data analysis. On the basis of previous investigations of sentence norms (Bloom \& Fischler, 1980; Lahar et al., 2004; Schwanenflugel, 1986), this sample size was deemed adequate.

Of the 377 participants, $83.6 \%$ were female $(n=315)$. The distribution of ethnicities was as follows: $62.3 \%$ white not of Hispanic origin $(n=235), 23.6 \%$ African American/African descent $(n=89)$, $2.7 \%$ Asian $(n=10), 2.9 \%$ Hispanic or Latino origin $(n=11), 1 \%$ native Hawaiian/Pacific Islander $(n=4)$, and $0.5 \%$ American Indian or Alaskan $(n=2)$. A percentage of participants $(6.9 \%, n=26)$ classified themselves as multicultural.

Additional data were collected on variables including class standing, level of education, handedness, and frequency of computer usage. The purpose of the inclusion of these variables was to provide additional descriptive data for future comparative studies. In terms of class standing, $40.8 \%$ of the sample classified themselves as college freshmen $(n=154), 26.8 \%$ as college sophomores $(n=101)$, $20.2 \%$ as college juniors $(n=76)$, and $11.1 \%$ as college seniors $(n=42)$; the additional $1.1 \%(n=4)$ were graduate students.

In addition, handedness and computer proficiency were assessed, with $88.6 \%$ reporting being right-handed $(n=344)$ and $93.1 \%$ re- porting daily computer usage $(n=351)$. Another $6.1 \%$ of the sample reported using a computer five to six times per week $(n=23)$, and the remaining $0.8 \%$ reported using a computer once or twice per week or less $(n=3)$. The high rate of computer usage confirms expectations that the present sample would not have difficulty logging online, using the online survey format, and reading the sentences to complete this study.

Generation of sentence contexts. Our research team generated approximately 460 sentences that were likely to produce a highly probable single-word response. Modeled after the methodology of Bloom and Fischler (1980), sentences were constructed according to five criteria. First, the sentence had to be grammatically correct with the addition of a single word, and each sentence had to have at least one other word that would result in a grammatically correct sentence (other than the highly probable response). Obvious clichés were avoided and single-word associations were avoided. Singleword associations are those for which, regardless of the context of the entire sentence, two words are likely to be reported together (e.g., "He felt the part-time job was his real bread and ...").

Additionally, Bloom and Fischler (1980) limited their sentences to 6 to 8 words. However, in an effort to obtain a large sample of high-constraint sentences, we extended this range slightly. Aborn, Rubenstein, and Sterling (1959) reported that context reaches its maximum effect between 5 and 10 words. Therefore, our sentences ranged from 6 to 11, with the majority being 10 words or fewer. Refer to Table 1 for representative sentences.

The initial sentence samples were piloted by obtaining sentence completion words from members of the research team, who had not generated the actual sentence in question, and from small samples (fewer than 20 per setting) of additional volunteers. Sentences resulting in more than one response from 2 or more people were eliminated. From these sentences, 398 were selected for inclusion in the large-scale survey.

In order to validate our methods and establish the reliability of our sample, we included 100 high-constraint sentences from the original 1980 norms established by Bloom and Fischler. Fifty of these were drawn from Bloom and Fischler's VU list, and another 50 were drawn from their LU list. Sentences had to meet the high-constraint probability of .67 or higher, and their high-probability cloze words had to be composed of a single syllable. These 100 sentences were interspersed randomly with the 398 new sentences created for the present study, for a total set of 498 sentences. The survey was pretested to ensure that completion time was within the reasonable limits for expected undergraduate attention span, and to minimize the possibility of participant fatigue.

Procedure. The final survey, consisting of 498 sentences, was administered individually and anonymously via Inquisite, a commercially available online survey program. The online survey could be taken in the participant's place of choice and allowed participants to self-pace and take rest breaks as they deemed appropriate. The survey began with a statement describing the purpose of the study,

Table 1

Cloze Probabilities for Representative Sentences

\begin{tabular}{rlcccc}
\hline \multicolumn{1}{c}{ Sentence Stem } & Response & $\begin{array}{c}\text { Present } \\
(2010)\end{array}$ & Response & $\begin{array}{c}\text { Bloom and } \\
\text { Fischler } \\
\text { (1980) }\end{array}$ \\
\hline 1. She could tell he was mad by the tone of his & voice & .99 & & \\
2. She went to the bakery for a loaf of & bread & .98 & & \\
3. Bob proposed and gave her a diamond & ring & .98 & & \\
4. The dentist recommends brushing your teeth twice a & day & .98 & neck & .96 (LU) \\
5. He loosened the tie around his & neck & .97 & & \\
6. Dan was asked to be the new coach of the & team & .97 & & \\
7. They paid for their meals but forgot to leave a & tip & .97 & & \\
8. To pay for tuition she took out two student & loans & .97 & & \\
9. She didn't have her watch so she asked for the & time & .97 & & \\
10. Sherry had to read lips because she was & deaf & .97 & & & \\
\hline
\end{tabular}

Note-The full set of 498 sentences is available in the online supplement. 
and participants were asked to acknowledge informed consent via buttonpress. They then began the survey, providing the single word they thought would best complete each sentence. Upon completion of the study, participants received a small amount of research credit that could be applied toward one of their psychology classes. The survey and research protocol were reviewed and approved by a review board. All ethical guidelines were adhered to in accordance with federal law.

Analyses. All responses were manually inspected to account for morphological variations. Words with one missing or one additional letter (e.g., cladss or clas), transposed letters (e.g., calss), capitalized letters (e.g., CLASS), or accidental punctuation (e.g., class.) were counted toward the intended word (e.g., class). Similar words or synonyms (e.g., bright and light) were not counted toward the intended word (e.g., glow). Missing data from individual sentences were below $5 \%$ and were not thought to significantly influence the results.

Cloze probability, as previously defined, was calculated for each sentence. For sentences that yielded low cloze values-meaning that numerous primary responses were generated - descriptives statistics were run, and the mean number of responses generated was examined.

As a measure of reliability, correlations were then performed between the cloze values obtained in the present investigation and those obtained by Bloom and Fischler (1980) for both their VU and LU sentence sets.

\section{Results and Discussion}

Cloze value characteristics for the entire sentence set. Frequencies were run for each sentence. A total of 400 of the 498 sentences met the definition of high cloze probability, with proportions ranging from .67 to .99 . Refer to Table 1 for a list of representative sentences. (The full sentence set is available electronically in the supplemental materials.) Of the 400 high cloze probability sentences, 321 were from the new set constructed for the present investigation. An additional 79 sentences meeting the definition of high cloze probability were from the original Bloom and Fischler (1980) sentences, with proportions ranging from .67 to .97 . Thirty-one of these sentences were from their VU set, and 48 were from their LU set. The remaining 21 of the 100 Bloom and Fischler sentences tested in the present study resulted in cloze probabilities in the medium and low ranges. The majority of these sentences fell within the medium cloze probability range rather than the low range (17 and 4, respectively). Note that, as expected, a majority of sentences (all but 2) not meeting the definition of high cloze probability came from the VU list. All sentences in the low cloze probability range were obtained from the VU set.

Eighty-six of the 498 sentences met the medium cloze probability criterion, with a range of .34 to .66 . This included 69 of the newly constructed sentences, as well as an additional 15 from the VU list and 2 from the LU list. The remaining 12 sentences were in the low cloze probability range of 0 to .33 , with 8 from the newly constructed set, 4 from the VU set, and none from the LU set. The lowest obtained value was for a new sentence, with a cloze probability of .17 .

Sentences with medium and low cloze probability generated a large number of different final word responses. Whereas high cloze probability sentences resulted in an average of 14 different word responses, the medium cloze sentences yielded an average of 25 different words and the low cloze probability sentences produced an average of 56 distinct responses. The reduced constraint of the low cloze sentences thus resulted in an increase in both number and variation of responses across subjects (e.g., for the sentence "Dillinger once robbed that ...", rather than the majority of responses being limited to "bank," responses varied from "store," "person," "man," to "woman," etc.).

Reliability and verification of sentence materials. As a measure of the reliability of the sentence construction criteria originally utilized by Bloom and Fischler (1980), correlations were performed for the reported cloze values obtained across the present sample and Bloom and Fischler's norms for the VU and LU sentence sets. Both samples correlated significantly at the $p<.001$ level for the VU sentence set $(r=.61)$ and for the LU sentence set $(r=.48)$. We have no explanation why the LU set would be somewhat less strongly correlated than the VU set. Regardless, the correlations obtained here are within the range of those observed by Lahar et al. (2004) for high cloze probability sentences. Comparing only the 25 sentences with the highest cloze probability, their correlations ranged from .53 to .84 across four age groups. We included a wider range of cloze probability sentences and still observed highly significant correlations between our cloze probabilities and those obtained by Bloom and Fischler.

The significantly high correlation between responses obtained in both samples for the same items provides evidence for the reliability of the cloze probabilities obtained here. Experiment 2 was designed to provide further evidence for the validity of the newly constructed sentences.

\section{EXPERIMENT 2}

As previously discussed, the N400 ERP component is thought to reflect contextually based semantic processing. Sentences ending in an unexpected word (given the semantic context) elicit a larger N400 response (greater amplitude) relative to expected words (Kutas \& Hillyard, 1980, 1983). Therefore, our newly constructed high cloze probability sentences should result in lower N400 amplitudes when completed with the normed sentence-final word relative to another word of the same linguistic category. Experiment 2 was designed to test this prediction.

\section{Method}

Participants. An independent sample of 33 participants was initially obtained from both psychology classes at a university in Virginia and from the local community. One participant did not meet the hearing screening of $30 \mathrm{~dB}$ or lower hearing level (HL) for pure tones from 500 to $8000 \mathrm{~Hz}$. This individual was not included in any subsequent analysis.

The remaining 32 individuals, ranging in age from 18 to 77 years $(M=43.3$ years, $S D=21)$, were included in the analysis. In the present sample, $68.8 \%$ were female $(n=22)$. The distribution of ethnicities was as follows: $87.5 \%$ white not of Hispanic origin $(n=28), 9.4 \%$ African American/African descent $(n=3)$, and 2.7\% Asian $(n=1)$.

Sentence materials. The 400 sentences determined to have high cloze probability in Experiment 1 were used to construct two sets containing 200 sentences each. Half of the sentences in each set were randomly selected to be altered to end in a word other than 
the most frequent response (cloze word) obtained in Experiment 1. For these sentences (100 in each set) an alternative word from the same linguistic category (noun) was chosen and substituted. These incongruent sentences were interspersed with the 100 congruent sentences (those ending in the expected high cloze probability word) in a randomized order with the constraint that no more than four of either type could be presented in succession.

Sentences were digitally recorded in a fluent stream by a female speaker whose first and main language was English. Sentences were spoken with normal conversational inflection, intonation, prosody, and amplitude level intended to approximate 60 to 65 decibels using an A-weighted scale (dBA). Digitally recorded sentences were then edited, amplified, and attenuated using a commercially available sound editing software program, Cooledit, for consistent length and intensity (dBA). Consistent intensity for sentences was verified by examining the visual running average of each word in a sentence using an A-weighted scale and a slow time setting on a digital Bruel and Kjaer 2238 mediator sound level meter, as recommended by Tschopp, Beckenbauer, and Harris (1991).

Sentences were presented via computer as an auditory processing task with a modified version of an existing Visual Basic program and stereophonic headphones with active noise reduction. Participants were instructed to respond by keypress, "1" for "yes" if the sentence seemed congruent, or " 3 " for "no" if the sentence seemed incongruent.

ERP recording procedures. EEG activity was recorded from 12 sites using the International 10-20 placement system with silversilver chloride electrodes. Frontal sites included F3, Fz, and F4; central sites included $\mathrm{C} 3, \mathrm{Cz}$, and $\mathrm{C} 4$; and parietal sites included $\mathrm{P} 3, \mathrm{Pz}$, and $\mathrm{P} 4$. Electrode sites were referenced online to linked earlobes and grounded to the central hairline on the equipotential plane of the cortical surface. Ocular artifacts were monitored via electrodes placed on the forehead (Fp1) and below the infraorbital ridge and orbital ridge of the right eye (VEOG1, VEOG2). Participants were asked to keep their eyes open and to be as relaxed as possible to lessen the likelihood of involuntary eye movements. However, explicit requests to monitor eye blinking were avoided, to refrain from introducing a secondary task within the experiment. Trials contaminated with eye movements, muscle activity, or amplifier-blocking artifacts were rejected offline prior to averaging. Artifacts with amplitude greater or less than $75 \mu \mathrm{V}$ were rejected.

Data were collected using a NuAmps Model 7181 Digital EEG amplifier and Neuroscan 4.2 software. Electrode impedances were kept below $5 \Omega$. Data were processed through a DC amplifier and were continuously digitized at $250 \mathrm{~Hz}$. A $60-\mathrm{Hz}$ notch filter was used to reduce artifacts. The N400 component was defined as the peak negativity occurring between 250 and $500 \mathrm{msec}$ after the onset of the sentence-final word (Kutas \& Hillyard, 1980, 1983). The N400 was averaged across event type and adjusted for duration of the sentence-final word, and the resulting latencies and amplitudes within the defined window were calculated offline.

Procedure. Written informed consent was obtained, then participants filled out the demographic questionnaire and completed the audiometric hearing test. Participants were then provided with an overview of the nature of EEG and recording electrophysiological activity. Electrodes were then attached to each participant's scalp. Participants practiced the auditory sentence task with an abbreviated sentence set containing 20 sentences (a random ordering of 10 congruent and 10 incongruent). Participants then performed the experimental trials by performing the auditory task with one of the two sets of 200 sentences in counterbalanced order. Following the first set, they rested briefly, then performed the task while listening to the other set. Their EEG was simultaneously recorded throughout.

Analyses. A 2 (congruency: incongruent vs. congruent) $\times 2$ (sentence list: List A vs. List B) repeated measures multivariate ANOVA with dependent measures being N400 amplitude at electrode sites $\mathrm{Fz}, \mathrm{Cz}$, and $\mathrm{Pz}$ was utilized to examine the neurophysiological response to expected versus unexpected sentence-final words.

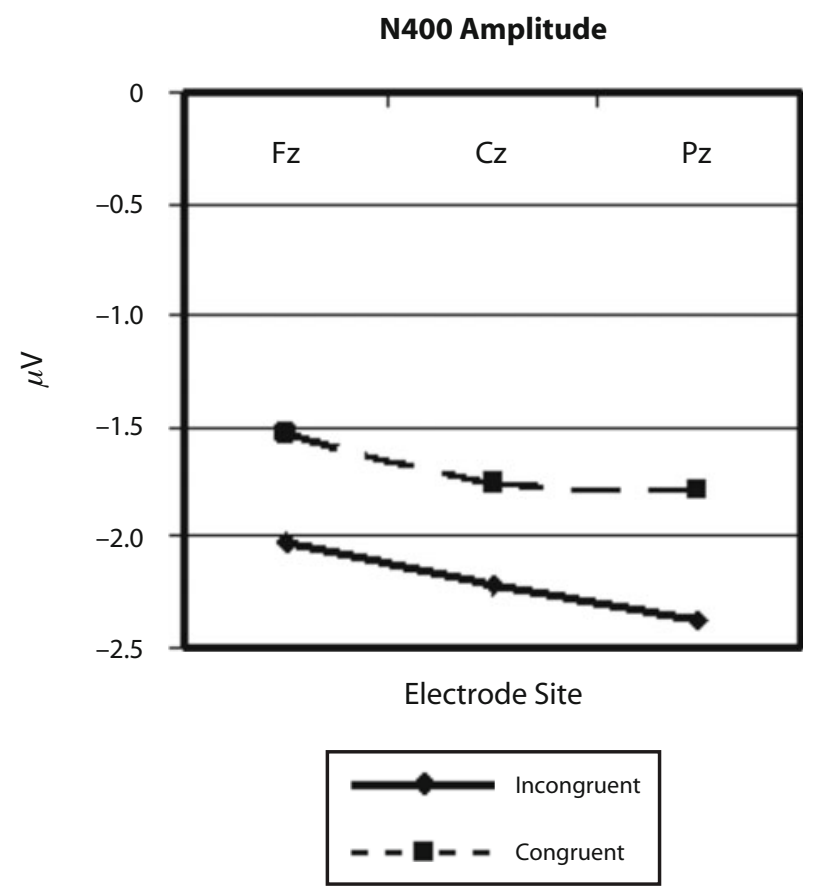

Figure 1. Grand average N400 amplitude for sentence-final words at electrode sites $\mathrm{Fz}, \mathrm{Pz}$, and $\mathrm{Cz}$, as a function of congruent and incongruent sentence-final word endings.

\section{Results and Discussion}

A significant main effect for congruency $[F(3,29)=$ $\left.5.57, \eta_{\mathrm{p}}^{2}=.37\right]$ was observed. As predicted and illustrated in Figure 1, N400 amplitude was greater for unexpected (incongruent) sentence-final words relative to expected (congruent) final words at each electrode site. These neurophysiological results obtained from a sample of participants different from those in Experiment 1 provide converging evidence for the validity of the newly constructed high cloze probability sentences.

\section{GENERAL DISCUSSION}

The aim of the present study was to expand the existing database of high-constraint, high cloze probability sentences using the criteria established by Bloom and Fischler (1980). In total, an additional 321 high-constraint sentences were validated and normed. An additional 79 sentences from a selection of Bloom and Fischler's original lists were also validated as having high cloze probability. Together, results of the present investigation provide a database of 400 high cloze probability sentences that have been normed with an undergraduate student population. This database is expected to be of use in a number of future experimental endeavors.

A high degree of consistency was obtained between responses from the present sample and the original Bloom and Fischler (1980) sample for a majority of the 100 sentences examined in the present investigation. As expected, more sentences from their low uncertainty (LU) list (48 of 50) achieved our high cloze criterion of .67. From the varied uncertainty (VU) set, 31 out of 50 sentences met 
the high cloze criterion. Also as expected, the VU set led to a greater number of alternative responses relative to the strong primary responses obtained with the LU set. The present completion norms also closely match the results observed by Lahar et al. (2004), who also observed a high degree of consistency with the Bloom and Fischler results for the LU but not VU items.

The ERP investigation in Experiment 2 provided further validation of the completion norms for the 400 high cloze probability sentences. As expected, high cloze probability sentences elicited an amplified N400 component when finished with an unexpected or incongruent word (a word other than the high-probability completion norm).

Standardized sentence materials are useful in many domains of research, including cognitive, neuroscience, neuropsychological, audiological, and gerontological studies. They provide a reliable way to assess speech processing as well as underlying sensory and cognitive processes and their associated brain mechanisms. For example, sentence sets with high cloze values allow an accumulation of context that will facilitate the elicitation of robust N400. Utilization of language materials such as the present set can also assist in understanding the relative influence of sensory and cognitive processes, and the susceptibility of these processes to age-related changes (Baldwin, 2007; Baldwin \& Struckman-Johnson, 2002). The expanded database of high cloze probability sentences presented here will allow the use of standardized materials to facilitate comparisons across investigations for many years to come.

\section{AUTHOR NOTE}

We acknowledge Lisa Peterson for assistance with data collection, Patricia Block for making the sentence recordings used in Experiment 2, Ian Reagan for assistance with constructing the program used to present the sentences in Experiment 2, and the National Institutes of Health, National Institute on Aging (Grant 1R03 AG023881-01 awarded to the second author) for financial support. Correspondence concerning this article should be addressed to C. L. Baldwin, Department of Psychology, ARCH Lab, MS 3F5, George Mason University, 4400 University Drive, Fairfax, VA 22030-4444 (e-mail: cbaldwi4@gmu.edu).

\section{REFERENCES}

Aborn, M., Rubenstein, H., \& Sterling, T. D. (1959). Sources of contextual constraint upon words in sentences. Journal of Experimental Psychology, 57, 171-180.

BALDWIN, C. L. (2007). Cognitive implications of facilitating echoic persistence. Memory \& Cognition, 35, 774-780.

Baldwin, C. L., \& Struckman-Johnson, D. L. (2002). Impact of speech presentation level on cognitive task performance: Implications for auditory display design. Ergonomics, 45, 61-74.

Bloom, P. A., \& FischleR, I. (1980). Completion norms for 329 sentence contexts. Memory \& Cognition, 8, 631-642.

Bradshaw, J. L. (1984). A guide to norms, ratings, and lists. Memory \& Cognition, 12, 202-206.

Coulson, S., Urbach, T. P., \& Kutas, M. (2006). Looking back: Joke comprehension and the space structuring model. Humor: International Journal of Humor Research, 19, 229-250.

Federmeier, K. D., Mclennan, D. B., De Ochoa, E., \& Kutas, M. (2002). The impact of semantic memory organization and sentence context information on spoken language processing by younger and older adults: An ERP study. Psychophysiology, 39, 133-146.

Federmeier, K. D., van Petten, C., Schwartz, T. J., \& Kutas, M. (2003). Sounds, words, sentences: Age-related changes across levels of language processing. Psychology \& Aging, 18, 858-872.

Kutas, M., \& Hillyard, S. A. (1980). Reading senseless sentences: Brain potentials reflect semantic incongruity. Science, 207, 203-205.

Kutas, M., \& Hillyard, S. A. (1983). Event-related brain potentials to grammatical errors and semantic anomalies. Memory \& Cognition, 11, 539-550.

Kutas, M., \& Hillyard, S. A. (1984). Brain potentials during reading reflect word expectancy and semantic association. Nature, 307, $161-163$.

Lahar, C. J., Tun, P. A., \& Wingfield, A. (2004). Sentence-final word completion norms for young, middle-aged, and older adults. Journals of Gerontology, 59B, P7-P10.

SCHWANENFlugel, P. J. (1986). Completion norms for final words of sentences using a multiple production measure. Behavior Research Methods, Instruments, \& Computers, 18, 363-371.

Schwanenflugel, P. J., \& LaCount, K. L. (1988). Semantic relatedness and the scope of facilitation for upcoming words in sentences. Journal of Experimental Psychology: Learning, Memory, \& Cognition, 14, 344-354.

Stine-Morrow, E. A. L., Miller, L. M. S., \& Nevin, J. A. (1999). The effects of context and feedback on age differences in spoken word recognition. Journals of Gerontology, 54B, P125-P134.

TAYLOR, W. L. (1953). "Cloze procedure": A new tool for measuring readability. Journalism Quarterly, 30, 415-433.

Traxler, M. J., \& Foss, D. J. (2000). Effects of sentence constraint on priming in natural language comprehension. Journal of Experimental Psychology: Learning, Memory, \& Cognition, 26, 1266-1282.

Tschopp, K., BeCKenBaUer, T., \& HARRIS, F. P. (1991). Objective measures of sentence level with respect to loudness. Audiology, 30, 113122

Van Berkum, J. J. A., Brown, C. M., Zwitserlood, P., Kooljman, V. \& HagoorT, P. (2005). Anticipating upcoming words in discourse: Evidence from ERPs and reading times. Journal of Experimental Psychology: Learning, Memory, \& Cognition, 31, 443-467.

\section{SUPPLEMENTAL MATERIALS}

The full set of 498 stimuli discussed in this article, along with the sentence completion norms, may be downloaded from http://brm .psychonomic-journals.org/content/supplemental.

(Manuscript received December 9, 2009; revision accepted for publication February 13, 2010.) 